\title{
Carrier localization effect in polarized InGaN multiple quantum wells
}

\author{
A. Žukauskas ${ }^{* 1}$, K. Kazlauskas ${ }^{1}$, G. Tamulaitis ${ }^{1}$, J. Mickevičius ${ }^{1}$, S. Juršėnas ${ }^{1}$, G. Kurilčik ${ }^{1}$, \\ S. Miasojedovas ${ }^{1}$, M. Springis ${ }^{2}$, I. Tale ${ }^{2}$, Yung-Chen Cheng ${ }^{3}$, Hsiang-Chen Wang ${ }^{3}$, \\ Chi-Feng Huang ${ }^{3}$, and C. C. Yang ${ }^{3}$ \\ ${ }^{1}$ Institute of Materials Science and Applied Research, Vilnius University, \\ Sauletekio al. 9, Build. III, 10222 Vilnius, Lithuania \\ 2 Institute of Solid State Physics, University of Latvia, Kengaraga iela 8, Riga 1063, Latvia \\ ${ }^{3}$ Graduate Institute of Electro-Optical Engineering, National Taiwan University, \\ 1 Roosevelt Road, Sec. 4, Taipei, Taiwan
}

Received 12 July 2004, accepted 26 September 2004 Published online 8 February 2005

PACS 73.21.Fg, 78.55.Cr, 78.67.De

Carrier localization effects in polarized InGaN/GaN multiple quantum wells (MQWs) were investigated as a function of well width, $d$, and In content, $x$. Using photoreflectance (PR), photoluminescence (PL), PL excitation (PLE), selective excitation of PL, PL excitation power, and time-resolved PL spectroscopy, the dominance of the localization effect against the built-in field effect on carrier recombination dynamics in $\mathrm{In}_{x} \mathrm{Ga}_{1-x} \mathrm{~N}$ MQWs of different well width $(d=2.0-4.0 \mathrm{~nm}, x \approx 0.15)$ and In content $(x \approx 0.22-0.27$, $d=2.5 \mathrm{~nm}$ ) was revealed. Based on the modeling of the PL spectra by Monte Carlo simulation of exciton hopping and the spectroscopic reference provided by PR, increased In content and well width were found to increase the band potential fluctuations and carrier localization depth. The density of localized states deduced from the simulation was found to be in a fair agreement with the PLE data. The built-in field strength in InGaN QWs containing $15 \%$ of In was estimated to be of $0.5 \mathrm{MV} / \mathrm{cm}$.

(C) 2005 WILEY-VCH Verlag GmbH \& Co. KGaA, Weinheim

1 Introduction InGaN/GaN multiple quantum wells (MQW) are widely used in mass fabrication of short-wavelength light-emitting devices. However, there is still much controversy about the role of localization and built-in field in the emission efficiency of these devices. Numerous microscopy observations [1-4] evidence an inhomogeneous distribution of indium in InGaN alloy, what is believed to cause localization of nonequilibrium carriers and an enhancement of the performance of InGaN-based light-emitting devices trough preventing the carriers from reaching nonradiative recombination sites [5]. On the other hand, the rate of radiative recombination in InGaN MQWs can be reduced by separation of the electron and hole wavefunctions by built-in electric field due to spontaneous and piezoelectric polarization at the well-barrier interfaces (the quantum confined Stark effect, QCSE). However, both the effects (localization and the QCSE) result in similar features of carrier recombination dynamics (a red shift of the PL peak, which decreases with the carrier density, and a longer carrier decay time) and they are difficult to distinguish between.

In this paper, we report on distinguishing between the localization and built-in field effects in InGaN/GaN MQWs with different well width and In content based on photoreflectance (PR), photoluminescence (PL), PL excitation (PLE), selective excitation of PL (SEPL), PL excitation power (PLEP), and time-resolved PL (TRPL) spectroscopy. The localization depth in a series of InGaN QWs was evaluated by analyzing of exciton hopping using Monte Carlo simulation.

"Corresponding author: e-mail: arturas.zukauskas@ff.vu.It, Phone: +370 5236 6059, Fax: +370 52366059 
2 Experimental Two sets of samples containing 5-nm thick InGaN quantum wells separated by 9-nm thick GaN barriers were deposited on GaN/sapphire templates using MOCVD technique. In the first set, the quantum well width was gradually changed from 2 to $4 \mathrm{~nm}$ at a fixed In content (15\%), whereas in the second set, the In content was varied from $22 \%$ to $27 \%$ at a fixed well width $(2.5 \mathrm{~nm})$.

$15 \mathrm{~mW} \mathrm{He}-\mathrm{Cd}$ laser $(325 \mathrm{~nm}$ ) was used for continuous-wave PL excitation, whereas chopped laser radiation and a halogen lamp were used in PR measurements. PLE spectra were measured by using a 150-W Xe lamp. For PLEP and SEPL measurements, a tunable organic-dye laser pumped by the frequency quadrupled Q-switched YAG:Nd laser (pulse duration $10 \mathrm{~ns}$ ) was used. In TRPL, the fourth harmonic of an actively-passively mode-locked YAG:Nd laser was used and the temporal resolution (20 ps) was provided by an optical Kerr shutter. The collected light was dispersed by a double monochromator and recorded by a photomultiplier. The measurements were carried out in the temperature range of $10-300 \mathrm{~K}$.

3 Results and discussion The PR and low-excitation PL spectra for $\operatorname{In}_{0.15} \mathrm{Ga}_{0.85} \mathrm{~N} / \mathrm{GaN}$ MQWs with the different thickness of the wells are shown in Fig. 1. The spectral separation between the PL peak and the fundamental optical transition energy (indicated by arrows), estimated from the PR spectra by fitting it with the standard Lorentzian functional form, is seen to increase for thicker QWs. Also, the PL and PR features are broadened with increasing the well width. The well-width dependences of the PL and PR energies are shown in Fig. 2. Since the built-in field reduces the PL and PR energies by the same extent, their difference (the Stokes shift) yields solely the carrier (exciton) localization depth. The Stokes shift is seen to enhance with the well width, what is probably due to an enhancement of the band potential fluctuations, which the carriers are localized at.

Numerical solution of the Schrödinger equation was used to fit the well-width dependence of the experimentally obtained fundamental optical transition energy. The dashed line in Fig. 2 shows the best fit of the experiment with theory achieved for the built-in field strength $F=0.5 \mathrm{MV} / \mathrm{cm}$. Solid line shows the calculated transition energies in the rectangular QWs $(F=0)$. The energy difference between the solid and dashed lines gives the quantitative estimate of the red shift due to the QCSE. This red shift is seen to be of about 5 times smaller than that due to the localization effect (the energy difference between the dashed and dotted lines).

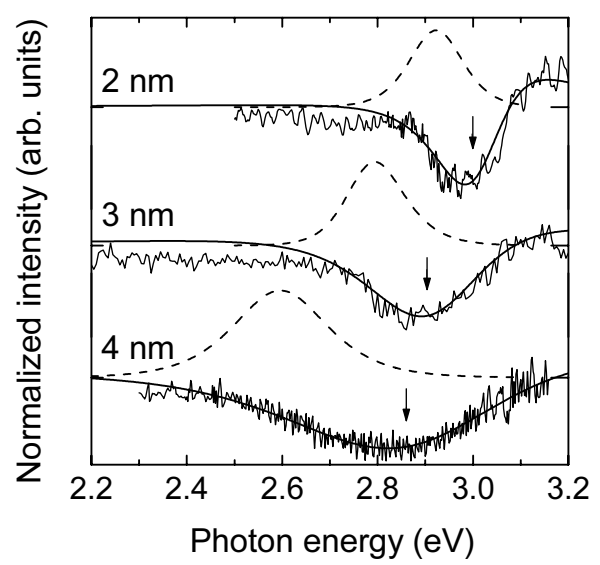

Fig. 1 PL (dashed lines) and PR (solid lines) spectra in $\mathrm{In}_{0.15} \mathrm{Ga}_{0.85} \mathrm{~N} / \mathrm{GaN}$ MQWs with different thickness of the wells (indicated). The PR spectra are fitted with Lorentzian lineshape functional form. Arrows indicate the energy of the fundamental optical transition.

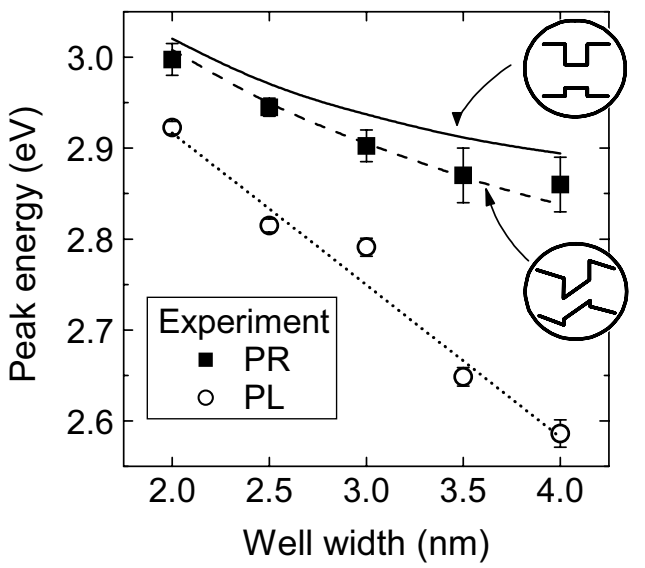

Fig. 2 Well-width dependence of the fundamental optical transition energy (squares) and PL peak energy (circles) in $\mathrm{In}_{0.15} \mathrm{Ga}_{0.85} \mathrm{~N} / \mathrm{GaN}$ MQWs, as estimated from the PR and PL spectra, respectively. Dashed line, the calculated transition energy in the tilted wells $(F=0.5 \mathrm{MV} / \mathrm{cm})$; solid line, same for the rectangular wells. Dotted line is a guide for the eye. 


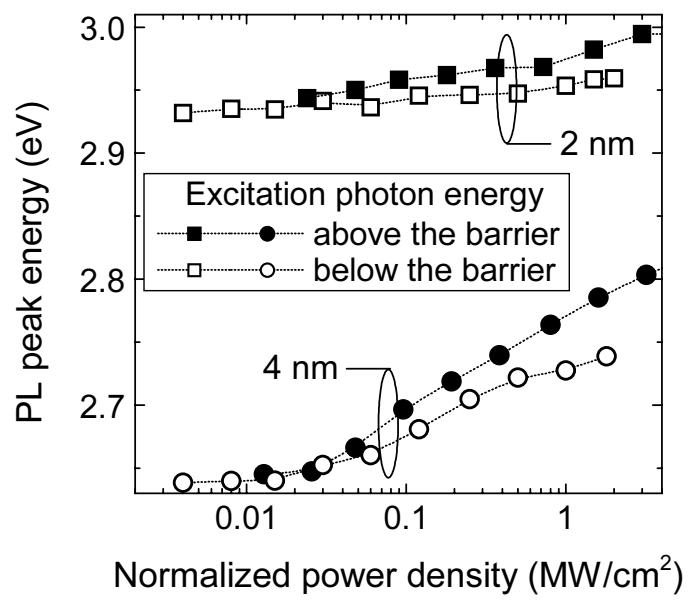

Fig. 3 Excitation power density dependence of the PL band peak position in 2-nm (squares) and 4-nm thick (circles) $\mathrm{In}_{015} \mathrm{Ga}_{085} \mathrm{~N}$ quantum wells. Filled points, the excitation photon energy above the bandgap energy of the barriers; open points, the excitation photon energy below the bandgap energy of the barriers.

Figure 3 shows dynamics of the PL peak with the excitation power density measured in 2- and 4-nm thick QWs for two excitation photon energies. In either case of the excitation photon energy, the PL peak exhibits a blue shift, which is larger for the thicker wells, what can be attributed to both the localizedstates filling and the built-in field screening effect. However, the observed blue shift is much larger than that expected for screening of the built-in electric field, and therefore it should be attributed mainly to band-tail filling. Moreover, this assumption is supported by that a larger blue shift is observed under excitation with higher-energy photons. Upon relaxing from barriers down to the wells, carriers most likely fall into localized states within In-rich regions, situated at the barrier-well interface and containing less indium than those formed in the wells. Formation of such interface regions was proved by highresolution transmission electron microscopy [2] and explained by indium inter-diffusion to GaN barriers. As a result, an enhanced distribution in localization energy within the tail states causes a larger blue shift.

In InGaN QWs with different In content, the peak position and linewidth of the PL spectra measured at low excitation power exhibited a well-established S- and W-shaped temperature behavior, which is known to be a signature of exciton hopping through the localized states $[6,7]$. The observed $\mathrm{W}$-shaped dependences of the PL linewidth, shown by points in Fig. 4, were described quantitatively by using Monte Carlo simulation of exciton hopping in a double-scaled potential profile (lines in Fig. 4) [7]. The scale (the standard deviation) of the band potential fluctuations within the In-rich regions, $\sigma$, and the dispersion in the average band gap energy of the regions (the inhomogeneous broadening), $\Gamma$, were found to increase from 31 to $38 \mathrm{meV}$ and from 29 to $47 \mathrm{meV}$, respectively, with an increase of In content from $22 \%$ to $27 \%$. Moreover, TRPL data obtained under high excitation conditions, which ensure complete screening of built-in fields, demonstrated an increase of the spontaneous emission decay time with an increase of In content, what also points out an enhancement of the band potential fluctuations.

Simultaneously with the W-shaped temperature dependence of the linewidth, the Stokes shift between the average exciton energy and the PL peak was deduced from the Monte Carlo simulation. By using the simulated values of the Stokes shift and the measured PL peak positions, a reconstruction of the temperature dependence of the exciton energy in the QWs was performed. Arrows in Fig. 5 show the reconstructed exciton energy for InGaN/GaN MQWs containing 22\%, 25\%, and $27 \%$ of In collated with the PLE spectra (thin lines) at $77 \mathrm{~K}$. Bold lines in Fig. 5 depict the density of localized states deduced from the Monte Carlo simulation with the inhomogeneous broadening taken into account. One can see that the peaks of the density of localized states coincide with the kinks on the low-energy wing of the PLE spectra. This provides for an additional support for the model of deep localization of carriers and excitons in the MQWs under investigation. 


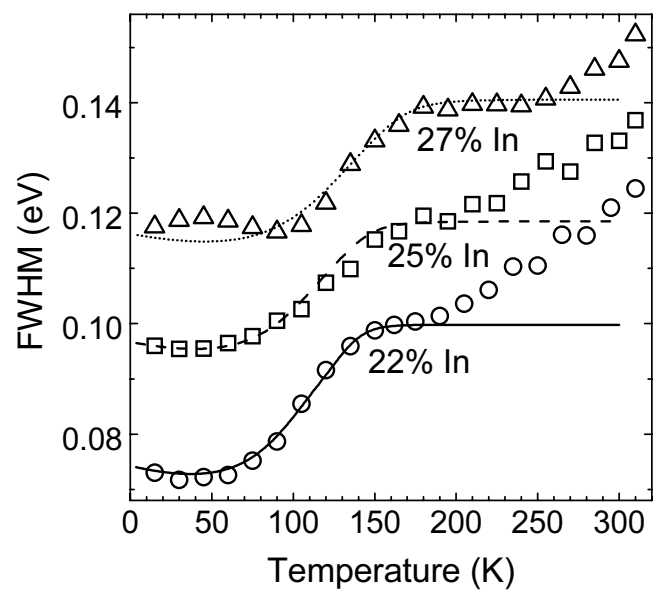

Fig. 4 Temperature dependences of the measured (points) and Monte Carlo-simulated (lines) dependences of full width at half maximum of the PL bands in $2.5 \mathrm{~nm}$-thick InGaN MQWs with different In content (indicated).

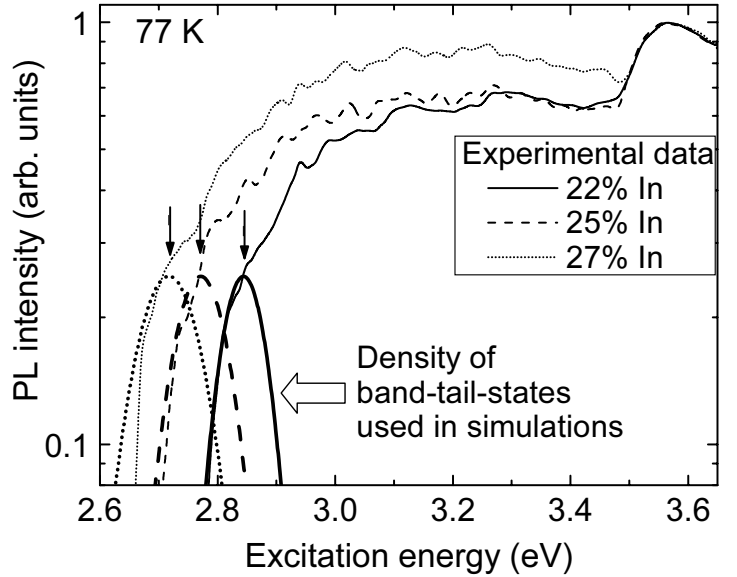

Fig. 5 PLE spectra of 2.5 nm-thick InGaN/GaN MQWs with different In content measured at $77 \mathrm{~K}$ (thin lines). Bold lines show inhomogeneously broadened density of band-tail-states used in simulations. Arrows indicate the exciton energy obtained from the simulation.

4 Conclusion The governing role of localization effect on carrier recombination dynamics was revealed in a series of InGaN/GaN MQWs with different well width and indium content. The small built-in field $(0.5 \mathrm{MV} / \mathrm{cm})$ in $\mathrm{In}_{0.15} \mathrm{Ga}_{0.85} \mathrm{~N} / \mathrm{GaN}$ MQWs, as estimated from the PR data, was demonstrated to have a minor influence on the Stokes shift of the emission energy. An increase of the well width and indium content was found to increase the band potential fluctuations, and consequently, the localization depth. The band potential profile roughness within In-rich regions and the dispersion of the average bandgap energy among these regions were quantified in terms of Monte Carlo simulation of exciton hopping and were shown to be in agreement with the PLE data.

Acknowledgements This research was partially supported by the joint Lithuanian-Latvian-Taiwan grant. A. Ž. and G. T. acknowledge the Lithuanian Ministry of Education and Science for their Fellowship.

\section{References}

[1] Y. Narukawa, Y. Kawakami, M. Funato, S. Fujita, S. Fujita, and S. Nakamura, Appl. Phys. Lett. 70, 981 (1997).

[2] Y.-S. Lin, K.-J. Ma, C. Hsu, S.-W. Feng, Y.-C. Cheng, C.-C. Liao, C. C. Yang, C.-C. Chou, C. -M. Lee, and J.I. Chyi, Appl. Phys. Lett. 77, 2988 (2000).

[3] S. Chichibu, K. Wada, and S. Nakamura, Appl. Phys. Lett. 71, 2346 (1997).

[4] J. Kim, K. Samiee, J. O. White, J.-M. Myoung, and K. Kim, Appl. Phys. Lett. 80, 989 (2002).

[5] S. Chichibu, T. Sota, K. Wada, and S. Nakamura, J. Vac. Sci. Technol. B 16, 2204 (1998).

[6] S. D. Baranovskii, R. Eichmann, and P. Thomas, Phys. Rev. B 58, 13081, (1998).

[7] K. Kazlauskas, G. Tamulaitis, A. Žukauskas, M. A. Khan, J. W. Yang, J. Zhang, G. Simin, M. S. Shur, and R. Gaska, Appl. Phys. Lett. 83, 3722 (2003). 